\title{
Baryon structure from Lattice QCD
}

\author{
C. Alexandrou ${ }^{1,2}$ \\ 1 Department of Physics, University of Cyprus, \\ P.O. Box 20357, Nicosia, CY-1678 Cyprus \\ 2 Computation-based Science and Technology Research Center, \\ Cyprus Institute, 20 Kavafi Street Nicosia 2121, Cyprus
}

\begin{abstract}
We present recent lattice results on the baryon spectrum, nucleon electromagnetic and axial form factors, nucleon to $\Delta$ transition form factors as well as the $\Delta$ electromagnetic form factors. The masses of the low lying baryons and the nucleon form factors are calculated using two degenerate flavors of twisted mass fermions down to pion mass of about $270 \mathrm{MeV}$. We compare to the results of other collaborations. The nucleon to $\Delta$ transition and $\Delta$ form factors are calculated in a hybrid scheme, which uses staggered sea quarks and domain wall valence quarks. The dominant dipole nucleon to $\Delta$ transition form factor is also evaluated using dynamical domain wall fermions. The transverse density distributions of the $\Delta$ in the infinite momentum frame are extracted using the form factors determined from lattice QCD.
\end{abstract}

PACS numbers: 11.15.Ha, 12.38.Gc, 12.38.Aw, 12.38.-t, 14.70.Dj

\section{INTRODUCTION}

During the last five years we have seen tremendous progress in dynamical lattice simulations using a number of different fermion discretization schemes with quark masses reaching closer to the physical pion mass. Many collaborations are contributing to this progress. The European Twisted Mass Collaboration (ETMC) is using twisted mass fermions (TMF), which provide an attractive formulation of lattice QCD that allows for automatic $\mathcal{O}(a)$ improvement, infrared regularization of small eigenvalues and fast dynamical simulations [1, 2]. Automatic $\mathcal{O}(a)$ improvement is obtained by tuning only one parameter requiring no further improvements on the operator level. A drawback of twisted mass fermions is the $\mathcal{O}\left(a^{2}\right)$ breaking of isospin symmetry, which is only restored in the continuum limit. In the baryon sector it has been shown that this isospin breaking is consistent with zero within our statistical accuracy by evaluating the mass difference between $\Delta^{++}\left(\Delta^{-}\right)$and $\Delta^{+}\left(\Delta^{0}\right)$ 3, 4, . This is in agreement with a theoretical analysis [5, 6] that shows potentially large $\mathcal{O}\left(a^{2}\right)$ flavor breaking effects to appear in the $\pi^{0}$-mass but to be suppressed in other quantities. A number of collaborations, as for example QCDSF [7], PACS-CS [8], BMW [9] and CERN [10] are using improved Clover fermions for their simulations. It is worth mentioning that PACS-CS has simulations very close to the physical pion mass albeit in a small volume, whereas the Wuppertal group recently calculated meson masses and the decay constants using $N_{F}=2+1$ configurations simulated at the physical pion mass [11]. A number of groups adopted a hybrid approach to compute hadronic matrix elements taking advantage of the efficient simulation and availability of staggered sea fermions produced by the MILC collaboration [12] and the chiral symmetry of domain wall fermions. The Lattice Hadron Physics Collaboration (LHPC) has been particularly active in producing results on a number of key observables [13, 14], some of which will be discussed in Sections IV and
V. A very promising recent development is the simulation of dynamical chiral fermions using large volumes and at small enough pions masses. The RBC-UKQCD collaboration is generating gauge configurations using $N_{F}=2+1$ domain wall fermions (DWF) [15], whereas the JLQCD Collaboration is producing dynamical configurations with two flavors of overlap fermions [16]. Most of the current simulations are done using volumes of spatial length $L$ such that $m_{\pi} L>3.5$ to keep finite volume effects small. The fact that simulations in the chiral regime are possible is to a large extend due to algorithmic improvements that yield better scaling behavior as the physical pion mass is approached. For a discussion on the scaling and a comparison among the different fermion discretization schemes see Ref.[17].

\section{HADRON SPECTRUM}

The masses of the lowest lying hadrons of a given set of quantum numbers are readily calculated by computing the two-point function at zero momentum: $C_{h}(t)=$ $\sum_{\mathbf{x}}\left\langle 0\left|J_{h}(\mathbf{x}, t) J_{h}^{\dagger}(0)\right| 0\right\rangle$. Choosing good interpolating fields and applying smearing techniques ensure ground state dominance at short time separation $t$ so that gauge noise is kept small [18]. In Figs. 1 and 2 we compare recent results on the low lying baryon spectrum using dynamical twisted mass [3, 19] and clover fermions [8] and within the hybrid approach [20] (staggered sea and domain wall valence quarks). The level of agreement of lattice QCD results using a variety of fermion discretization schemes seen in Figs. 1 and 2 before taking the continuum limit or other lattice artifacts into account is quite impressive. Small discrepancies seen mainly in the decuplet masses can be attributed to lattice artifacts and a systematic analysis of these effects is performed by each collaboration before extracting the final continuum values. In particular results using staggered fermions may 


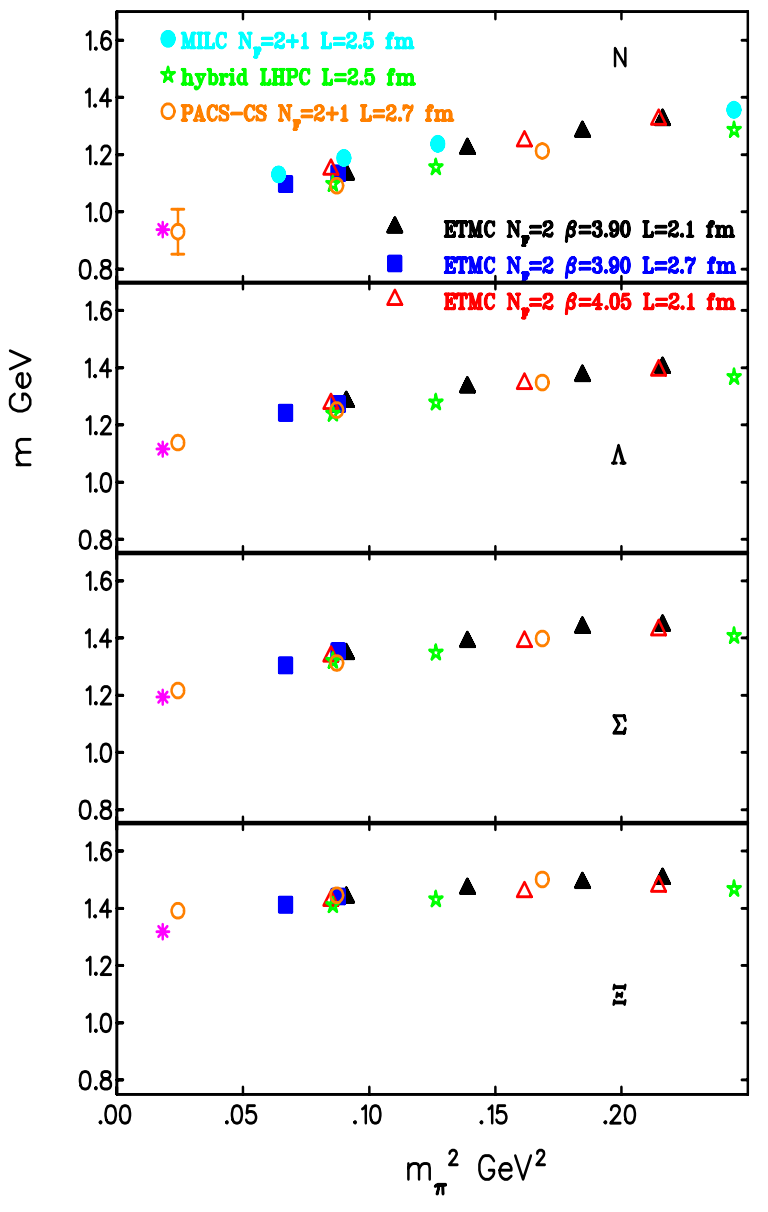

FIG. 1: Comparison of masses for the low lying octet baryons. Results using $N_{F}=2 \mathrm{TMF}$ are shown by the filled (black) triangles for $L=2.1 \mathrm{fm}$ and (blue) squares for $L=2.7 \mathrm{fm}$ with $a=0.089 \mathrm{fm}$ and with the open (red) triangles for $L=2.1 \mathrm{fm}$ and $a=0.070 \mathrm{fm}$. Results with the hybrid action are shown with the (green) asterisks for $a=0.124 \mathrm{fm}$ and results using $N_{F}=2+1$ Clover fermions with the open (orange) circles and $a=0.0907 \mathrm{fm}$. For the nucleon we also show results using $N_{F}=2+1$ staggered fermions (filled (light blue) circles). The physical masses are shown by the (purple) star.

suffer the most from cut-offs effects since the lattice used is rather coarse as compared to those using twisted mass and Clover fermions which have lattice spacings smaller than $0.1 \mathrm{fm}$.

Having reliable methods to extract the masses of the low lying hadrons one can investigate techniques for the extraction of the masses of excited states. A number of approaches exist. A commonly used method is based on the variational approach [21]: For a given $N \times N$ correlator matrix $C_{k n}(t)=\left\langle 0\left|J_{k}(t) J_{n}^{\dagger}(0)\right| 0\right\rangle$ one defines the $N$ principal correlators with $\lambda_{k}\left(t, t_{0}\right)$ as the eigenvalues of

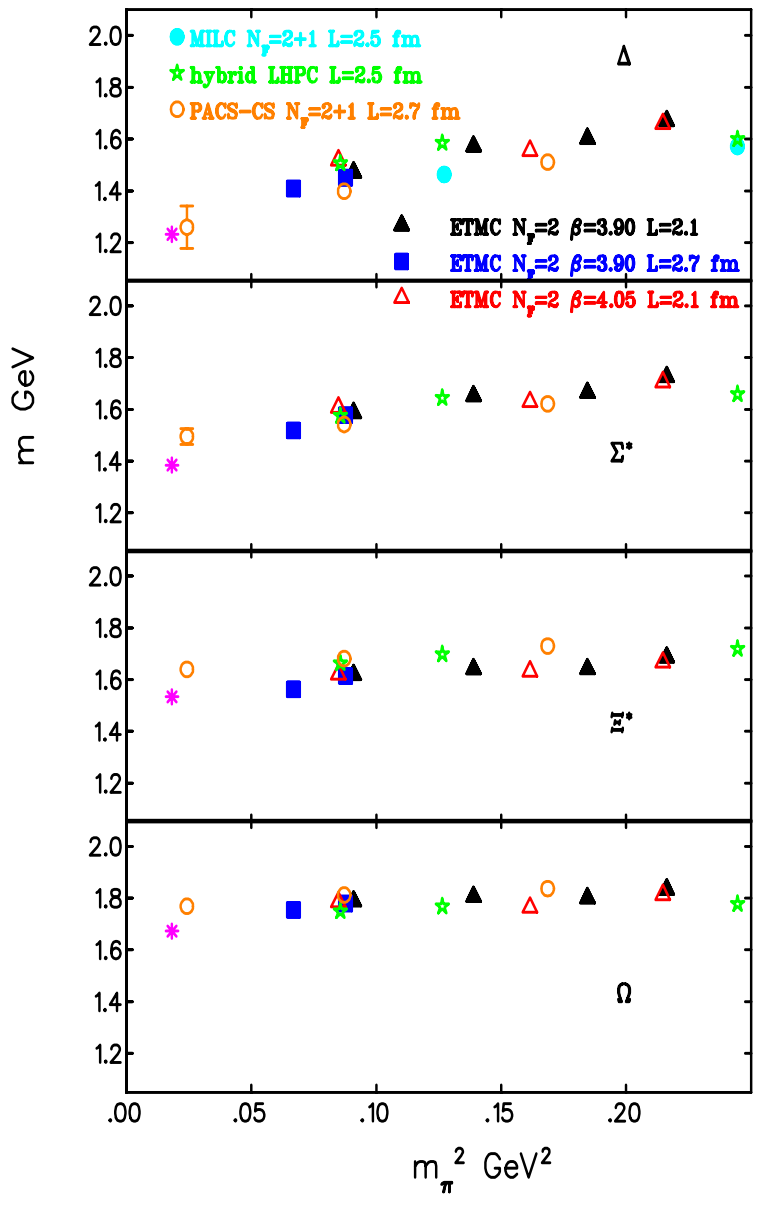

FIG. 2: Comparison of masses for the low lying decuplet baryons. The notation is the same as that of Fig. 1 .

$C\left(t_{0}\right)^{-1 / 2} C(t) C\left(t_{0}\right)^{-1 / 2}$, where $t_{0}$ is small. Since

$\lim _{t \rightarrow \infty} \lambda_{k}\left(t, t_{0}\right)=e^{-\left(t-t_{0}\right) E_{k}}\left(1+e^{-t \Delta E_{k}}\right), \quad k=1, \ldots, N$

the $N$ principal effective masses tend (plateau) to the $\mathrm{N}$ lowest-lying stationary-state energies of the hadrons with the same quantum numbers. It is crucial to use very good operators so noise does not swamp signal and construct spatially extended operators using smearing of the quark fields as well as applying link variable smearing. The use of a large set of appropriately constructed operators is also very important. Despite recent calculations using this method 22] the issue of the ordering of the Roper resonance as compared to the negative parity partner of the nucleon still remains unresolved. Maximum entropy methods have also be developed for the analysis of hadron two-point correlators and recent results can be found in Ref. 223]. A new method that relies solely on $\chi^{2}$ minimization with an unbiased evaluation of errors can be applied to extract the masses of the states on which the two-point correlator is sensitive on [24]. This method was applied to extract the excited states of the nucleon using local correlators that are easily produced in lattice simulations. For this study two interpolating fields are 

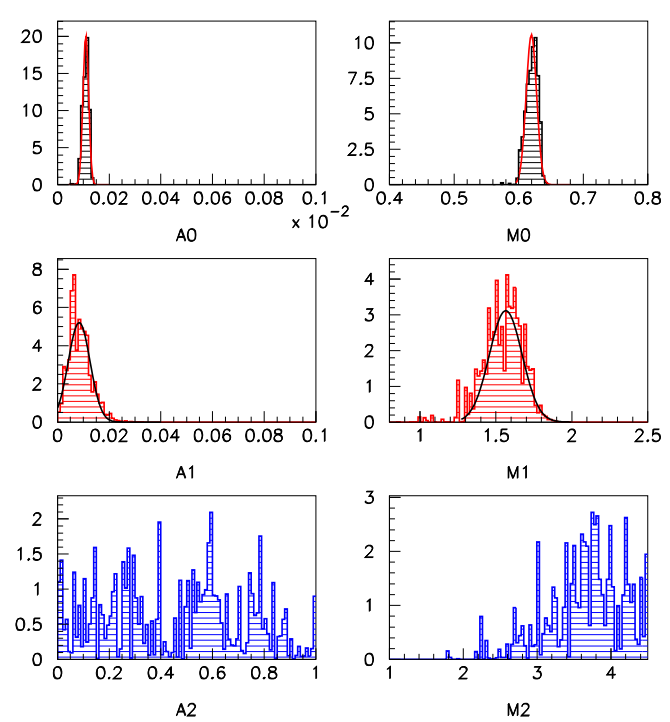

FIG. 3: Probability distributions for the amplitudes and masses in lattice units extracted from local correlators using $N_{F}=2$ Wilson fermions at pion mass $500 \mathrm{MeV}$ on a lattice of spatial length $1.8 \mathrm{fm}$ at $\beta=6.0 \mathrm{using} J_{N}$.
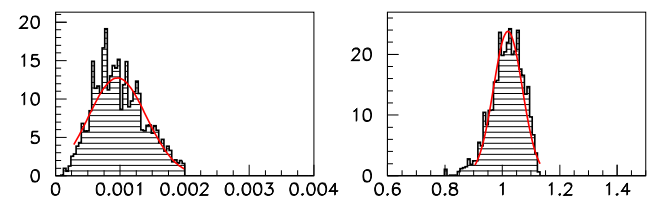

$\mathrm{AO}$

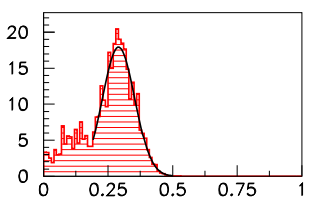

A1
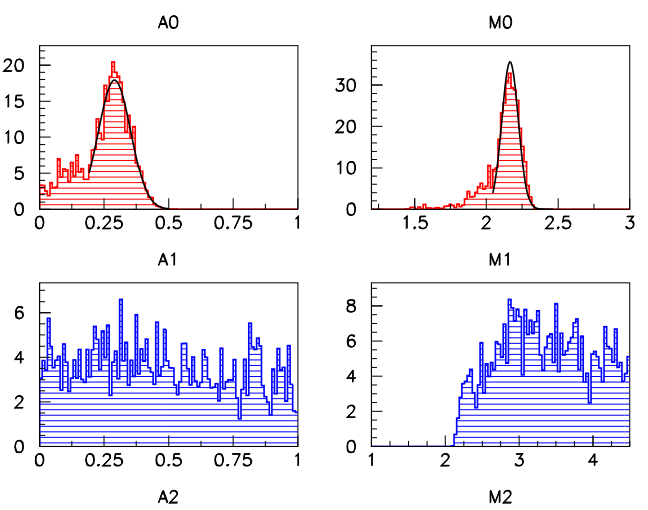

M1

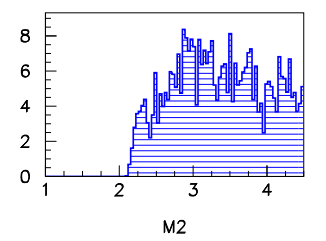

FIG. 4: As in Fig. 3 but using the interpolating field $J_{N}^{\prime}$.

considered:

$$
\begin{aligned}
& J_{N}(x)=\epsilon^{a b c}\left(u_{a} C \gamma_{5} d_{b}^{T}\right) u_{c} \\
& J_{N}^{\prime}(x)=\epsilon^{a b c}\left(u_{a}^{T} C d_{b}\right) \gamma_{5} u_{c} .
\end{aligned}
$$

As can be seen from the histograms shown in Figs. 3 and 4, one clearly identifies the first excited state in the positive parity channel of the nucleon using rather low quality data. In addition, we observe that the state of lowest mass that is present in the mass spectrum of the correlator computed with $J_{N}$ is absent when using $J_{N}^{\prime}$. Instead the correlator with $J_{N}^{\prime}$ has a lowest state that does not show up when using $J_{N}$. The conjecture is that this state is the Roper.

\section{FORM FACTORS}

To extract information on hadron structure one needs to calculate coupling constants, such as the nucleon axial charge $g_{A}$, the $\pi N$ and $\pi N \Delta$ coupling constants, form factors, moments of parton distribution functions and generalized form functions. In order to compute these quantities we need to calculate the relevant three-point functions, which, in addition to the forward propagator needed for the calculation of the masses, require the evaluation of the sequential propagator. The three-point function, related to the matrix element of the operator $\mathcal{O}$ between hadron states $\mid h^{\prime}>$ and $|h\rangle$, is given by

$$
\begin{gathered}
\left\langle G^{h^{\prime} \mathcal{O} h}\left(t_{2}, t_{1} ; \mathbf{p}^{\prime}, \mathbf{p} ; \Gamma\right)\right\rangle=\sum_{\mathbf{x}_{2}, \mathbf{x}_{1}} \exp \left(-i \mathbf{p}^{\prime} \cdot \mathbf{x}_{2}\right) \exp \left(+i \mathbf{q} \cdot \mathbf{x}_{1}\right) \\
\left\langle\Omega\left|\Gamma^{\beta \alpha} T\left[J_{h^{\prime}}^{\alpha}\left(\mathbf{x}_{2}, t_{2}\right) \mathcal{O}\left(\mathbf{x}_{1}, t_{1}\right) \bar{J}_{h}^{\beta}(\mathbf{0}, 0)\right]\right| \Omega\right\rangle
\end{gathered}
$$

where for $\mathcal{O}$ we consider the electromagnetic and axial currents. We use sequential inversions through the sink, which allows us to obtain the three-point function for any momentum transfer $\mathbf{q}$ and operator insertion but fixes the quantum numbers of the initial and final baryons.

\section{A. Nucleon Electromagnetic form factors}

The elastic nucleon electromagnetic form factors are fundamental quantities characterizing important features of neutron and proton structure that include their size, charge distribution and magnetization. An accurate determination of these quantities in lattice QCD is timely and important because of a new generation of precise experiments. The matrix element of interest is

$$
\begin{gathered}
\left\langle N\left(p^{\prime}, s^{\prime}\right)\left|A_{\mu}^{3}\right| N(p, s)\right\rangle=\left(\frac{m_{N}^{2}}{E_{N}\left(\mathbf{p}^{\prime}\right) E_{N}(\mathbf{p})}\right)^{1 / 2} \\
\bar{u}\left(p^{\prime}, s^{\prime}\right)\left[\gamma_{\mu} F_{1}\left(q^{2}\right)+\frac{i \sigma_{\mu \nu} q^{\nu}}{2 m_{N}} F_{2}\left(q^{2}\right)\right] u(p, s),
\end{gathered}
$$

where $p(s)$ and $p^{\prime}\left(s^{\prime}\right)$ denote initial and final momenta (spins) and $m_{N}$ is the nucleon mass, $F_{1}(0)=1$ for the proton and $F_{2}(0)$ measures the anomalous magnetic moment. These form factors are connected to the electric, $G_{E}\left(q^{2}\right)$, and magnetic, $G_{M}\left(q^{2}\right)$, Sachs form factors by the relations

$$
G_{E}\left(q^{2}\right)=F_{1}\left(q^{2}\right)+\frac{q^{2}}{\left(2 m_{N}\right)^{2}} F_{2}\left(q^{2}\right)
$$




$$
G_{M}\left(q^{2}\right)=F_{1}\left(q^{2}\right)+F_{2}\left(q^{2}\right) .
$$

To extract the nucleon matrix element from lattice measurements, we calculate, besides the three point function $G^{N j^{\mu} N}\left(t_{2}, t_{1} ; \mathbf{p}^{\prime}, \mathbf{p} ; \Gamma\right)$, the nucleon two-point function, $G^{N N}(t, \mathbf{p})$, and look for a plateau in the large Euclidean time behavior of the ratio

$$
\begin{gathered}
R\left(t_{2}, t_{1} ; \mathbf{p}^{\prime}, \mathbf{p} ; \Gamma ; \mu\right)=\frac{\left\langle G^{N j^{\mu} N}\left(t_{2}, t_{1} ; \mathbf{p}^{\prime}, \mathbf{p} ; \Gamma\right)\right\rangle}{\left\langle G^{N N}\left(t_{2}, \mathbf{p}^{\prime} ; \Gamma_{4}\right)\right\rangle} \\
{\left[\frac{\left\langle G^{N N}\left(t_{2}-t_{1}, \mathbf{p} ; \Gamma_{4}\right)\right\rangle\left\langle G^{N N}\left(t_{1}, \mathbf{p}^{\prime} ; \Gamma_{4}\right)\right\rangle\left\langle G^{N N}\left(t_{2}, \mathbf{p}^{\prime} ; \Gamma_{4}\right)\right\rangle}{\left\langle G^{N N}\left(t_{2}-t_{1}, \mathbf{p}^{\prime} ; \Gamma_{4}\right)\right\rangle\left\langle G^{N N}\left(t_{1}, \mathbf{p} ; \Gamma_{4}\right)\right\rangle\left\langle G^{N N}\left(t_{2}, \mathbf{p} ; \Gamma_{4}\right)\right\rangle}\right]^{1 / 2}} \\
t_{2}-t_{1} \gg 1, t_{1} \gg 1 \\
\Rightarrow
\end{gathered}
$$

where

$$
\left\langle G^{N N}(t, \mathbf{p} ; \Gamma)\right\rangle=\sum_{\mathbf{x}} e^{-i \mathbf{p} \cdot \mathbf{x}} \Gamma^{\beta \alpha}\left\langle\Omega\left|T J^{\alpha}(\mathbf{x}, t) \bar{J}^{\beta}(\mathbf{0}, 0)\right| \Omega\right\rangle .
$$

We use the lattice conserved electromagnetic current, $j^{\mu}(x)$, symmetrized on site $x$ and projection matrices for the Dirac indices

$$
\Gamma_{i}=\frac{1}{2}\left(\begin{array}{cc}
\sigma_{i} & 0 \\
0 & 0
\end{array}\right), \quad \Gamma_{4}=\frac{1}{2}\left(\begin{array}{ll}
I & 0 \\
0 & 0
\end{array}\right) .
$$

Throughout this work we use kinematics where the final nucleon state is produced at rest and therefore $\mathbf{q}=$ $\mathbf{p}^{\prime}-\mathbf{p}=-\mathbf{p}$. For the polarized matrix element one can construct an optimal linear combination for the nucleon sink, which in Euclidean time is given by

$$
\begin{aligned}
S_{m}(\mathbf{q} ; i)= & \sum_{k=1}^{3} \Pi\left(-\mathbf{q} ; \Gamma_{k} ; \mu=i\right)=\frac{C}{2 m_{N}}\left\{\left(p_{2}-p_{3}\right) \delta_{1, i}\right. \\
& \left.+\left(p_{3}-p_{1}\right) \delta_{2, i}+\left(p_{1}-p_{2}\right) \delta_{3, i}\right\} G_{M}\left(Q^{2}\right)
\end{aligned}
$$

with $Q^{2}=-q^{2}$. This construction provides the maximal set of lattice measurements from which $G_{M}\left(Q^{2}\right)$ can be extracted requiring one sequential inversion. No such improvement is necessary for the unpolarized matrix elements given by

$$
\Pi\left(\mathbf{0},-\mathbf{q} ; \Gamma_{4} ; \mu=i\right)=C \frac{q_{i}}{2 m_{N}} G_{E}\left(Q^{2}\right)
$$

and

$$
\Pi\left(\mathbf{0},-\mathbf{q} ; \Gamma_{4} ; \mu=4\right)=C \frac{E_{N}+m_{N}}{2 m_{N}} G_{E}\left(Q^{2}\right),
$$

which yield $G_{E}\left(Q^{2}\right)$ with an additional sequential inversion. $C=\sqrt{\frac{2 m_{N}^{2}}{E_{N}\left(E_{N}+m_{N}\right)}}$ is a factor due to the normalization of the lattice states.

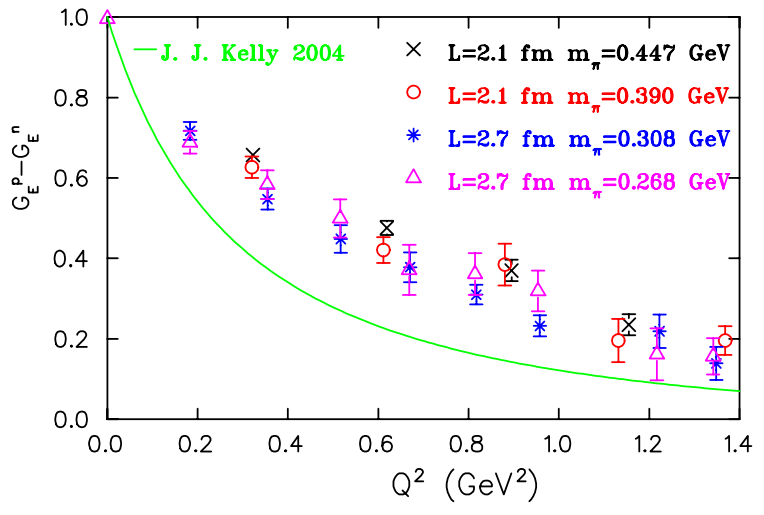

FIG. 5: Nucleon isovector electric form factor using $N_{F}=2$ TMF.

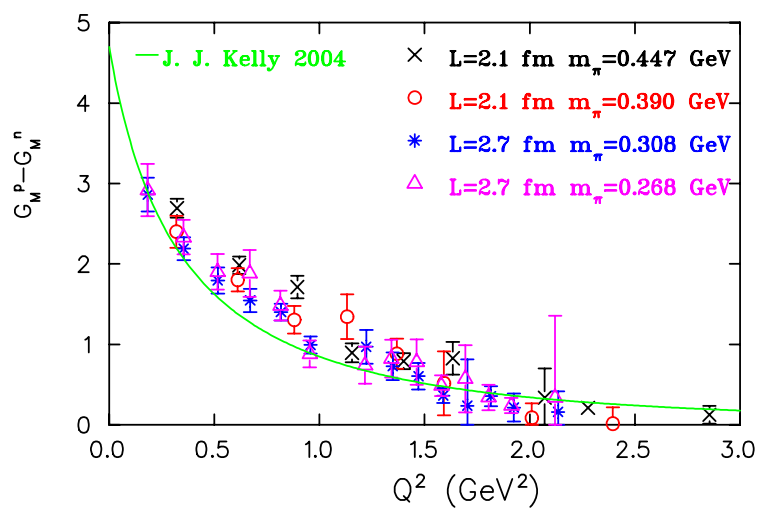

FIG. 6: Nucleon isovector magnetic form factor using $N_{F}=2$ TMF.

Besides using an optimal nucleon source, the other important ingredient in the extraction of the form factors is to take into account simultaneously in our analysis all the lattice momentum vectors that contribute to a given $Q^{2}$. This is done by solving the overcomplete set of equations

$$
P(\mathbf{q} ; \mu)=D(\mathbf{q} ; \mu) \cdot F\left(Q^{2}\right)
$$

where $P(\mathbf{q} ; \mu)$ are the lattice measurements of the ratio given in Eq. (6) having statistical errors $w_{k}$ and using the different sink types, $F=\left(\begin{array}{c}G_{E} \\ G_{M}\end{array}\right)$ and $D$ is an $M \times$ 2 matrix which depends on kinematical factors with $M$ being the number of current directions and momentum vectors contributing to a given $Q^{2}$. We extract the form factors by minimizing

$$
\chi^{2}=\sum_{k=1}^{N}\left(\frac{\sum_{j=1}^{2} D_{k j} F_{j}-P_{k}}{w_{k}}\right)^{2}
$$

using the singular value decomposition of $D$. The analysis described in this Section to extract $G_{E}\left(Q^{2}\right)$ and $G_{M}\left(Q^{@}\right)$ is also applied to the analysis of all form factors presented in this work.

The $\gamma N \rightarrow N$ transition contains isoscalar photon contributions. This means that disconnected loop di- 


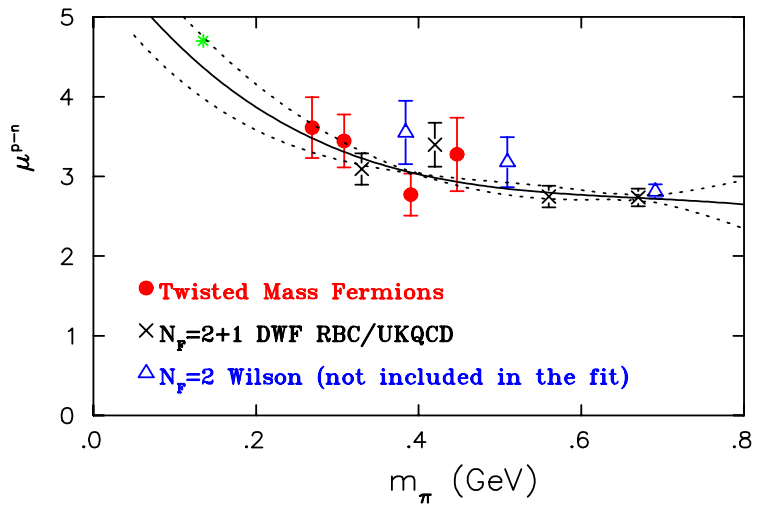

FIG. 7: Nucleon magnetic moment using $N_{F}=2$ TMF (filled circles), $N_{F}=2+1 \mathrm{DWF}$ [31] (crosses) and $N_{F}=2$ Wilson fermions [32](open triangles). The physical value is shown by the star.

agrams also contribute. These are generally difficult to evaluate accurately since the all-to-all quark propagator is required. In order to avoid disconnected diagrams, we calculate the isovector form factors. Assuming $S U(2)$ isospin symmetry, it follows that $\langle p|\left(\frac{2}{3} \bar{u} \gamma^{\mu} u-\right.$ $\left.\frac{1}{3} \bar{d} \gamma^{\mu} d\right)|p\rangle-\left\langle n\left|\left(\frac{2}{3} \bar{u} \gamma^{\mu} u-\frac{1}{3} \bar{d} \gamma^{\mu} d\right)\right| n\right\rangle=\langle p|\left(\bar{u} \gamma^{\mu} u-\right.$ $\left.\bar{d} \gamma^{\mu} d\right)|p\rangle$ and therefore by calculating the proton threepoint function related to the matrix element of the right hand side of the above relation we obtain the isovector nucleon form factors $G_{E}^{p}\left(q^{2}\right)-G_{E}^{n}\left(q^{2}\right)$ and $G_{M}^{p}\left(q^{2}\right)-G_{M}^{n}\left(q^{2}\right)$.

The results for the isovector electric and magnetic form factors using $N_{F}=2$ twisted mass fermions are shown in Figs. 5 and 6 25]. The lattice results on the electric form factor fall off slower as compared to a parametrization of the experimental data [26] shown by the solid line, whereas the magnetic form factor is closer to experiment. This is consistent with recent high accuracy results obtained by the LHP Collaboration [27] and the RBC-UKQCD Collaboration [28]. Fitting the magnetic form factor to a dipole form we extract $G_{M}(0)$, which determines the anomalous magnetic moment. We show its dependence on the pion mass in Fig. 7. Using chiral effective theory with explicit nucleon and $\Delta$ degrees of freedom to one-loop order the isovector anomalous magnetic moment 29], the Dirac and Pauli radii can be extrapolated to the physical point [18, 29, 30]. There are three fit parameters for the magnetic moment and the best fit to the twisted mass data with the associated error band is shown in Fig. 7 Multiplying the Pauli radius squared with the magnetic moment yields an expression with only one-parameter like the Dirac radius that can shift the curves but does not affect their slopes. As can be seen the physical magnetic moment is within the error band whereas for the radii results closer to the physical point are needed to check the predicted slope.
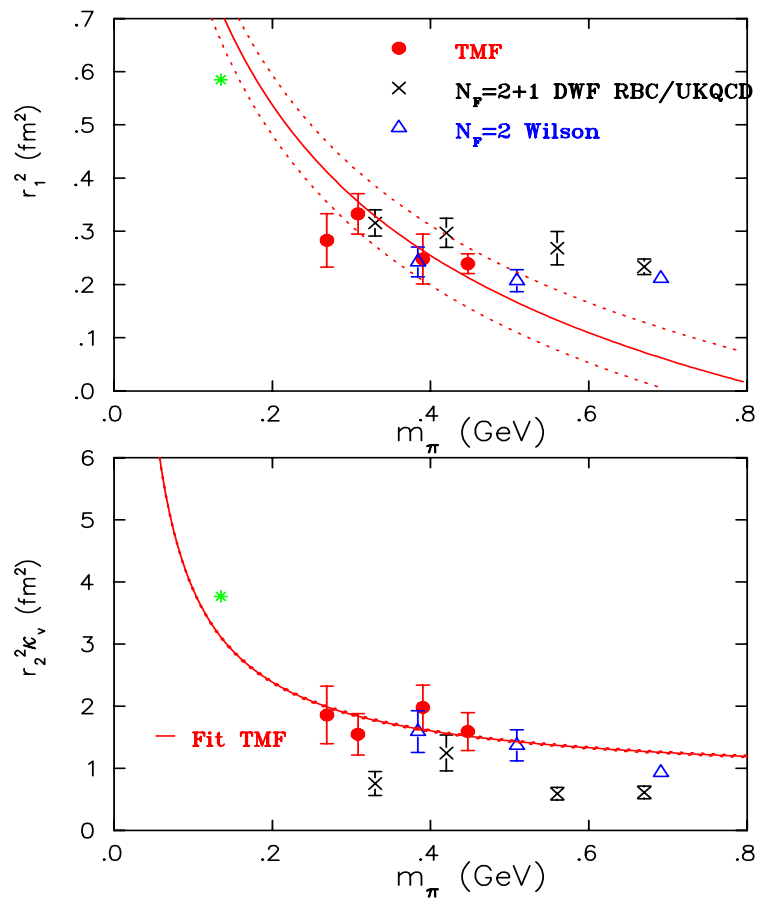

FIG. 8: The Dirac radius squared (top) and Pauli radius squared multiplied by the magnetic moment (bottom). The notation is the same as that of Fig. 7

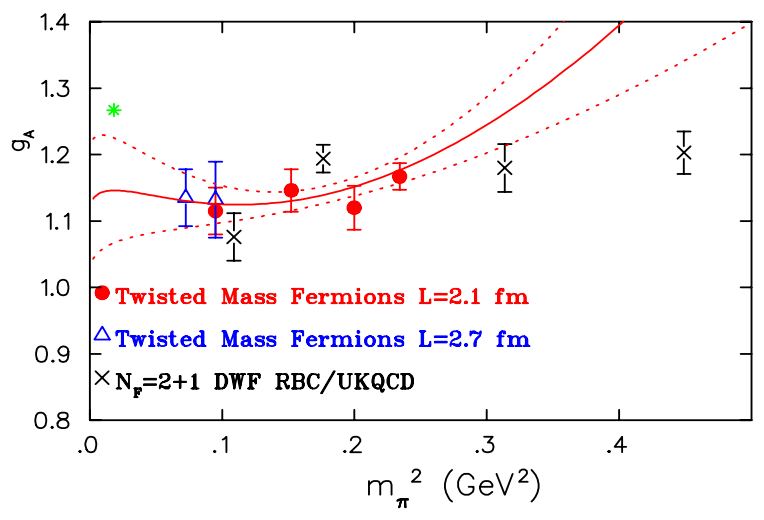

FIG. 9: The nucleon axial charge using $N_{F}=2 \mathrm{TMF}$ and $N_{F}=2+1$ DWF.

\section{B. Nucleon axial form factors}

The matrix element of the weak axial vector current between nucleon states can be written as

$$
\begin{aligned}
& \left\langle N\left(p^{\prime}, s^{\prime}\right)\left|A_{\mu}^{3}\right| N(p, s)\right\rangle=i\left(\frac{m_{N}^{2}}{E_{N}\left(\mathbf{p}^{\prime}\right) E_{N}(\mathbf{p})}\right)^{1 / 2} \\
& \bar{u}\left(p^{\prime}, s^{\prime}\right)\left[G_{A}\left(q^{2}\right) \gamma_{\mu} \gamma_{5}+\frac{q_{\mu} \gamma_{5}}{2 m_{N}} G_{p}\left(q^{2}\right)\right] \frac{\tau^{3}}{2} u(p, s)
\end{aligned}
$$

where the axial isovector current $A_{\mu}^{3}=\bar{\psi}(x) \gamma_{\mu} \gamma_{5} \frac{\tau^{3}}{2} \psi(x)$. 


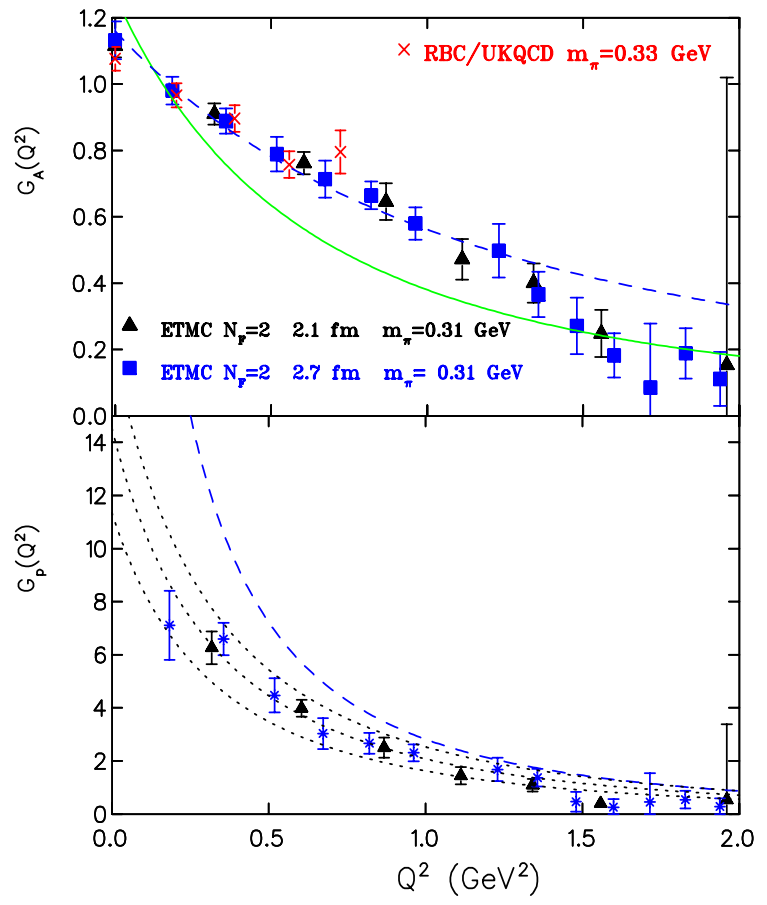

FIG. 10: Top: The nucleon axial form factors $G_{A}\left(Q^{2}\right)$ for $N_{F}=2 \mathrm{TMF}$ and $N_{F}=2+1 \mathrm{DWF}$. The dashed line is a dipole fit to the lattice data whereas the solid line to experiment. Bottom: $G_{p}\left(Q^{2}\right)$ for $N_{F}=2 \mathrm{TMF}$. The dashed line is predicted from $G_{A}\left(Q^{2}\right)$ and Eq. (15). The dotted lines is the best fit with the associated error band.

Having computed the nucleon electromagnetic form factors we can obtain the axial ones with no additional inversions 25, 32]. The advantage here is that only the connected diagram contributes. In addition at zero momentum transfer we obtain the nucleon axial charge $g_{A}$, a quantity that is very accurately measured experimentally. We show in Fig. 9 results obtained using $N_{F}=2$ twisted mass [25] and domain wall fermions [33]. The leading one-loop chiral perturbation theory result for $g_{A}$ in the small scale expansion [34] can be used to extrapolate lattice results to the physical point. Making a threeparameter fit to the twisted mass results we obtain the solid curve shown in Fig. 9 together with the error band determined by allowing the fit parameters to vary within a $\chi^{2}$ increase by one unit from the minimum. Note that this error band does not include uncertainties in the fixed parameters. We obtain, at the physical point, a value with a large error that is lower than the experimental value by an amount slightly larger than one standard deviation. Results closer to the physical pion mass are needed to reduce the error due to the chiral extrapolation.

The $Q^{2}$-dependence of the nucleon axial form factors $G_{A}\left(Q^{2}\right)$ and $G_{p}\left(Q^{2}\right)$ using $N_{F}=2$ twisted mass fermions is shown in Fig. 10, Our results for $G_{A}\left(Q^{2}\right)$ are in agreement with those obtained using $N_{F}=2+1$ domain wall fermions at a comparable value of the pion mass. The $Q^{2}$-dependence of $G_{A}\left(Q^{2}\right)$ can be well described by a dipole Ansatz $g_{0} /\left(Q^{2} / m_{A}^{2}+1\right)^{2}$ as shown by the dashed line. This is what is usually used to describe experimental data for $G_{A}\left(Q^{2}\right)$ where a value of $m_{A} \sim 1.1 \mathrm{GeV}$ is extracted for the axial mass. However the axial mass $m_{A}$ extracted from the lattice data is larger resulting in a slower fall off as compared to experiment shown by the solid line. Assuming pion pole dominance $G_{p}\left(Q^{2}\right)$ can be obtained in terms of $G_{A}\left(Q^{2}\right)$ as

$$
G_{p}\left(Q^{2}\right)=\frac{4 m_{N}^{2} / m_{\pi}^{2}}{1+Q^{2} / m_{\pi}^{2}} G_{A}\left(Q^{2}\right) .
$$

In Fig. 10 we show with the dashed line what pion pole dominance predicts if we use the fit determined from $G_{A}\left(Q^{2}\right)$. The error band shows the best fit to $G_{p}\left(Q^{2}\right)$ if we instead fit the strength and mass of the monopole in Eq. 15$]$

\section{N TO $\Delta$ TRANSITION FORM FACTORS}

The determination of the $\mathrm{N}$ to $\Delta$ electromagnetic and axial transition form factors requires the evaluation of the three-point function $\left\langle G_{\sigma}^{\Delta \mathcal{O} N}\left(t_{2}, t_{1} ; \mathbf{p}^{\prime}, \mathbf{p} ; \Gamma\right)\right\rangle$ with a new set of inversions, where for $\mathcal{O}$ we consider the electromagnetic and axial currents. The $\gamma^{*} N \Delta$ matrix element is given by

$$
\begin{gathered}
\left\langle\Delta\left(\mathbf{p}^{\prime}, s^{\prime}\right)\left|j_{\mu}\right| N(\mathbf{p}, s)\right\rangle=i \sqrt{\frac{2 m_{\Delta} m_{N}}{3 E_{\Delta}\left(\mathbf{p}^{\prime}\right) E_{N}(\mathbf{p})}} \bar{u}_{\sigma}\left(\mathbf{p}^{\prime}, s^{\prime}\right) \\
{\left[G_{M 1}\left(Q^{2}\right) K_{\sigma \mu}^{M 1}+G_{E 2}\left(Q^{2}\right) K_{\sigma \mu}^{E 2}+G_{C 2}\left(Q^{2}\right) K_{\sigma \mu}^{C 2}\right] u(\mathbf{p}, s) .}
\end{gathered}
$$

The evaluation of the two subdominant electromagnetic form factors $G_{E 2}\left(Q^{2}\right)$ and $G_{C 2}\left(Q^{2}\right)$, which are of primary interest as far as the question of deformation is concerned, require high accuracy. A lattice QCD calculation accurate enough to exclude a zero value to one standard deviation would point to deformation in the nucleon $/ \Delta$ system. This is particularly relevant given the fact that extraction of these form factors from experiment involves modeling and therefore a non-zero value from a first principles calculation even to one standard deviation is an important result. Optimized sinks are constructed to isolate the subdominant form factors [35] along the same lines as discussed for the polarized nucleon matrix element. In experimental searches for deformation, it is customary to quote the ratios of the electric and Coulomb quadrupole amplitudes to the magnetic dipole amplitude, EMR or $R_{E M}=-\frac{G_{E 2}\left(Q^{2}\right)}{G_{M 1}\left(Q^{2}\right)} R_{E M}$ and CMR or $R_{S M}=-\frac{|\vec{q}|}{2 m_{\Delta}} \frac{G_{C 2}\left(Q^{2}\right)}{G_{M 1}\left(Q^{2}\right)}$, in the rest frame of the $\Delta$. 


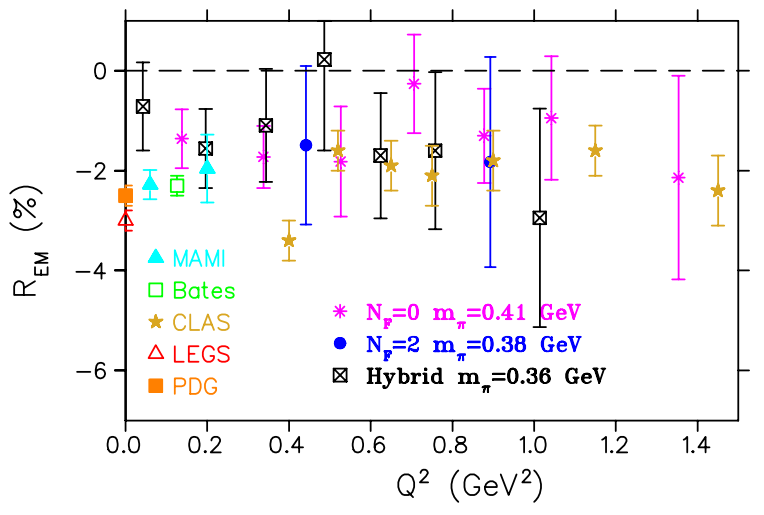

FIG. 11: The EMR calculated using quenched (stars) and dynamical (filled circles) Wilson fermions and in the hybrid approach (squares).

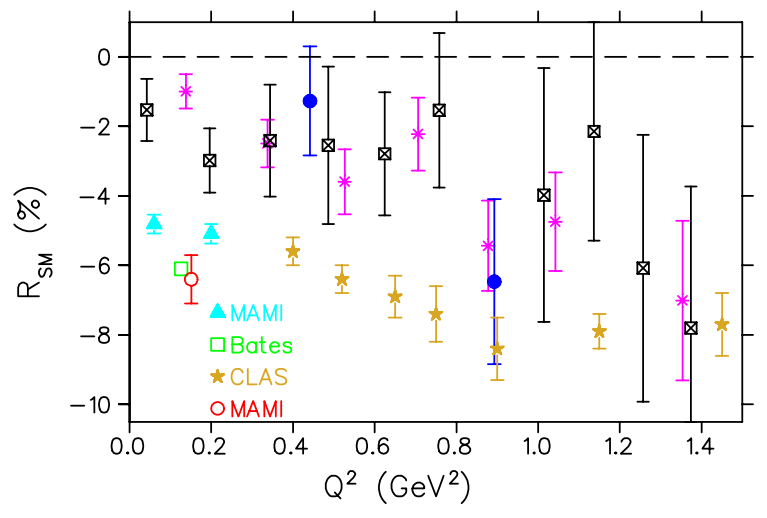

FIG. 12: The CMR calculated using Wilson fermions and in the hybrid approach.

Results on these ratios obtained using Wilson fermions and the hybrid action are shown in Figs. 11 and 12. Lattice results at low $Q^{2}$ are non-zero. The lattice values of CMR at small $Q^{2}$ are less negative than experiment. As the pion mas decreases lattice results tend to become more negative approaching experiment. Therefore one anticipates that for even smaller pion masses the discrepancy between lattice and experiment will be reduced since pion cloud effects are expected to make CMR more negative as we approach the physical regime [36].

In Fig. 13 we compare results for the dipole form factor $G_{M 1}\left(Q^{2}\right)$ obtained within the hybrid approach and using dynamical domain wall fermions at about the same mass. As can be seen there is very good agreement showing that results obtained within the non-unitary hybrid action are reliable.

The invariant proton to $\Delta^{+}$weak matrix element is expressed in terms of four transition form factors as

$$
<\Delta\left(p^{\prime}, s^{\prime}\right)\left|A_{\mu}^{3}\right| N(p, s)>=i \sqrt{\frac{2}{3}}\left(\frac{m_{\Delta} m_{N}}{E_{\Delta}\left(\mathbf{p}^{\prime}\right) E_{N}(\mathbf{p})}\right)^{1 / 2}
$$

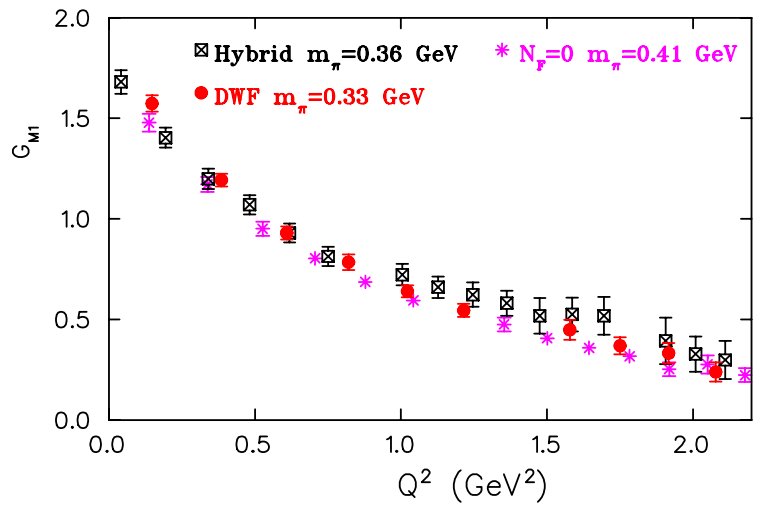

FIG. 13: $G_{M 1}\left(Q^{2}\right)$ in the hybrid approach and using $N_{F}=$ $2+1$ domain wall fermions. Quenched Wilson results are also included for comparison.

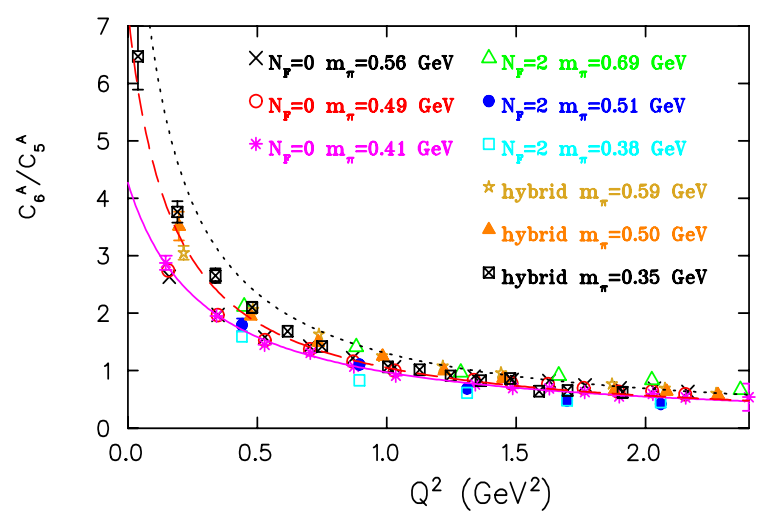

FIG. 14: The ratio of $N$ to $\Delta$ axial transition form factors $C_{6}^{A}\left(Q^{2}\right) / C_{5}^{A}\left(Q^{2}\right)$. The dotted line shows the pion pole dominance prediction for the hybrid case. The dashed and solid lines are fits to a monopole form for the hybrid and quenched results respectively.

$$
\begin{gathered}
\bar{u}_{\Delta^{+}}^{\lambda}\left(p^{\prime}, s^{\prime}\right)\left[\left(\frac{C_{3}^{A}\left(q^{2}\right)}{m_{N}} \gamma^{\nu}+\frac{C_{4}^{A}\left(q^{2}\right)}{m_{N}^{2}} p^{\prime \nu}\right)\left(g_{\lambda \mu} g_{\rho \nu}-g_{\lambda \rho} g_{\mu \nu}\right) q^{\rho}\right. \\
\left.+C_{5}^{A}\left(q^{2}\right) g_{\lambda \mu}+\frac{C_{6}^{A}\left(q^{2}\right)}{m_{N}^{2}} q_{\lambda} q_{\mu}\right] u_{P}(p, s)
\end{gathered}
$$

where $C_{5}^{A}\left(Q^{2}\right)$ and $C_{6}^{A}\left(Q^{2}\right)$, the dominant form factors, can be related assuming pion pole dominance like $G_{A}\left(Q^{2}\right)$ and $G_{p}\left(Q^{2}\right)$ are related in the nucleon case.

In Fig. 14 we plot the ratio $C_{6}^{A}\left(Q^{2}\right) / C_{5}^{A}\left(Q^{2}\right)$. The doted line shows the prediction assuming pion pole dominance after a dipole fit to the hybrid results on $C_{5}^{A}\left(Q^{2}\right)$ is performed. As in the nucleon case the ratio does not fall off as rapidly and a fit to a monopole form to the same hybrid results gives the dashed line. 


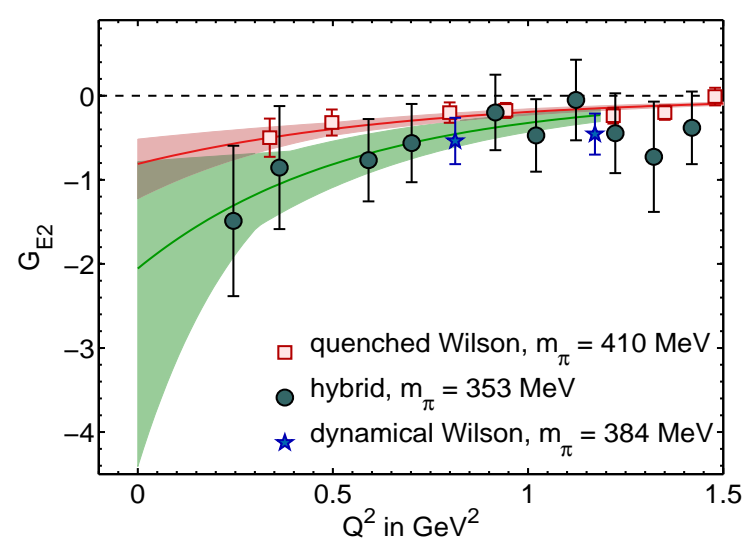

FIG. 15: The $Q^{2}$-dependence of $G_{E 2}\left(Q^{2}\right)$. The green (red) line and error band show a dipole fit to the mixed action (quenched ) results. The value of $G_{E 2}$, in units of $e /\left(2 m_{\Delta}^{2}\right)$, at $Q^{2}=0$ are $-0.810 \pm 291$ for the quenched calculation, $-0.87 \pm 67$ for $N_{F}=2$ Wilson case and $-2.06_{-2.35}^{+1.27}$ for the hybrid calculation.

\section{V. $\Delta$ ELECTROMAGNETIC FORM FACTORS AND DENSITY DISTRIBUTION}

Since the $\Delta(1232)$ decays strongly, experiments to measure its form factors are harder than for the $\mathrm{N}$ to $\Delta$ transition and yield less precise results. The $\Delta$ form factors can be computed using lattice QCD more accurately than can be currently obtained from experiment. The decomposition for the on shell $\gamma^{*} \Delta \Delta$ matrix element is given by

$$
\begin{array}{r}
\left\langle\Delta\left(p_{f}, s_{f}\right)\left|j_{\mathrm{EM}}^{\mu}\right| \Delta\left(p_{i}, s_{i}\right)\right\rangle=\mathcal{A} \bar{u}_{\sigma}\left(p_{f}, s_{f}\right) \mathcal{O}^{\sigma \mu \tau} u_{\tau}\left(p_{i}, s_{i}\right) \\
\mathcal{O}^{\sigma \mu \tau}=-g^{\sigma \tau}\left[a_{1}\left(q^{2}\right) \gamma^{\mu}+\frac{a_{2}\left(q^{2}\right)}{2 m_{\Delta}}\left(p_{f}^{\mu}+p_{i}^{\mu}\right)\right] \\
-\frac{q^{\sigma} q^{\tau}}{4 m_{\Delta}^{2}}\left[c_{1}\left(q^{2}\right) \gamma^{\mu}+\frac{c_{2}\left(q^{2}\right)}{2 m_{\Delta}}\left(p_{f}^{\mu}+p_{i}^{\mu}\right)\right],
\end{array}
$$

where $a_{1}\left(q^{2}\right), a_{2}\left(q^{2}\right), c_{1}\left(q^{2}\right)$, and $c_{2}\left(q^{2}\right)$ are known linear combinations of the electric charge form factor $G_{E 0}\left(q^{2}\right)$, the magnetic dipole form factor $G_{M 1}\left(q^{2}\right)$, the electric quadrupole form factor $G_{E 2}\left(q^{2}\right)$, and the magnetic octupole form factor $G_{M 3}\left(q^{2}\right)$. An optimized sink is constructed that isolated the subdominant electric quadrupole form factor [14, 37]. The results are shown in Fig. 15.

The electric quadrupole form factor is particularly interesting because it can be related to the shape of a hadron. Just as the electric form factor for a spin $1 / 2$ nucleon can be expressed precisely as the transverse Fourier transform of the transverse quark charge density in the infinite momentum frame [38], a proper field-theoretic interpretation of the shape of the $\Delta(1232)$ can be obtained by considering the quark transverse charge densities in this

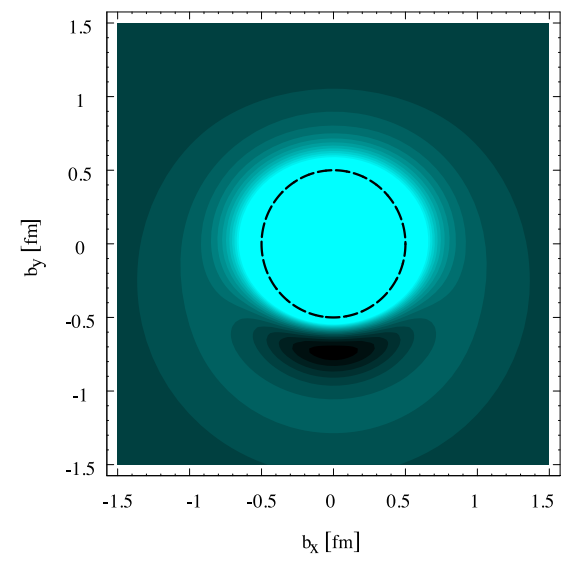

FIG. 16: Quark transverse charge density in a $\Delta^{+}$polarized along the $x$-axis, with $s_{\perp}=+3 / 2$. The light (dark) regions correspond with the largest (smallest) values of the density.

frame. Fig. 16 shows the transverse density $\rho_{T}^{\Delta}{ }_{s_{\perp}}$ for a $\Delta^{+}$with transverse spin $s_{\perp}=+3 / 2$ calculated from the fit to the quenched Wilson lattice results for the $\Delta$ form factors (which has the smallest statistical errors of the three calculations). It is seen that the $\Delta^{+}$quark charge density is elongated along the axis of the spin (prolate).

\section{CONCLUSIONS}

Lattice QCD simulations are now being carried out in the chiral regime by a number of collaborations. We have shown that there is agreement among recent lattice results using different fermion discretization schemes on the low lying baryon spectrum and the nucleon form factors. Recent results on the low lying hadron spectrum where lattice artifacts have been carefully examined are in perfect agreement with experiment providing validation of the lattice approach and QCD itself [9]. Furthermore we have shown that lattice QCD provides a framework for the computation of quantities that can not be accurately measured in experiment such as the $\Delta$ form factors providing valuable insight into the structure of such hadrons. We anticipate that other key hadronic quantities will be computed to sufficient accuracy and with lattice artifacts taken into account thereby providing direct comparison to experiment.

\section{Acknowledgments}

I would like to thank my close collaborators T. Korzec, G. Koutsou, J. W. Negele, C. N. Papanicolas, E. Stiliaris, A. Tsapalis and M. Vanderhaeghen for their valuable contributions. I would also like to thank my 
collaborators in the ETMC for their important contributions in various aspects of the work presented here and for a most enjoyable and fruitful collaboration. This work is partly supported by the Cyprus Research Promo- tion Foundation under contracts ПENEK/ENI $2 \mathrm{X} / 0505$ 39, EPYAN/0506/08 and KY-ГА/0907/11.
[1] R. Frezzotti, P. A. Grassi, S. Sint and P. Weisz [Alpha collaboration], JHEP 0108, 058 (2001).

[2] R. Frezzotti and G. C. Rossi, JHEP 0408, 007 (2004).

[3] C. Alexandrou et al. [ETM Collaboration], Phys. Rev. D 78, 014509 (2008).

[4] C. Alexandrou et al. [ETM Collaboration], PoS LAT2007, 087 (2007) arXiv:0710.1173 [hep-lat]].

[5] R. Frezzotti and G. Rossi, PoS LAT2007, 277 (2007).

[6] A. Shindler, Phys. Rept. 461, 37 (2008).

[7] M. Gockeler et al. [QCDSF Collaboration], PoS LAT2007, 041 (2007).

[8] S. Aoki et al. [PACS-CS Collaboration], Phys. Rev. D 79, 034503 (2009).

[9] S. Durr et al., Science 322, 1224 (2008).

[10] L. Del Debbio, L. Giusti, M. Luscher, R. Petronzio and N. Tantalo, JHEP 0702, 056 (2007).

[11] Y. Aoki et al. arXiv:0903.4155 [hep-lat]. [12]

[12] C. Aubin et al., Phys. Rev. D 70, 094505 (2004).

[13] Ph. Hagler et al. [LHPC Collaborations], Phys. Rev. D 77, 094502 (2008). arXiv:0705.4295 [hep-lat]].

[14] C. Alexandrou et al., Nucl. Phys. A 825, 115 (2009).

[15] P. Boyle [RBC Collaboration and UKQCD Collaboration], PoS LAT2007, 005 (2007); C. Allton et al. [RBCUKQCD Collaboration], Phys. Rev. D 78, 114509 (2008).

[16] S. Aoki et al. [JLQCD Collaboration], Phys. Rev. D 78, 014508 (2008).

[17] K. Jansen, arXiv:0810.5634 [hep-lat].

[18] C. Alexandrou, G. Koutsou, J. W. Negele and A. Tsapalis, Phys. Rev. D 74, 034508 (2006).

[19] V. Drach et al., PoS LAT2008, 123 (2008) arXiv:0905.2894 [hep-lat]]; C. Alexandrou et al., in preparation.

[20] A. Walker-Loud,et al., Phys Rev. D 79, 054502 (2009).

[21] C. Michael, Nucl. Phys. B259, 58 (1985); M. Lüscher and U. Wolff, Nucl. Phys. B339, 222 (1990).

[22] S. Basak et al., Phys. Rev. D 76, 074504 (2007); B. G. Lasscock et al., Phys. Rev. D 76, 054510 (2007); C. Gattringer et al., Phys. Rev. D 78, 034501 (2008); C. Morningstar, arXiv:0810.4448 [hep-lat].

[23] P. Lepage et al., Nucl. Phys. Proc. Suppl. 106 (2002) 12; C. Morningstar, Nucl. Phys. Proc. Suppl. 109A (2002) 185; T. Draperet al., arXiv:0810.5512 [hep-lat].

[24] C. Alexandrou, E. Stiliaris, C.N. Papanicolas, PoS LAT2008, arXiv:0810.3982

[25] C. Alexandrou et al. [ETMC], arXiv:0811.0724 [hep-lat].

[26] J. J. Kelly, Phys. Rev. C 70, 068202 (2004).

[27] J. D. Bratt et al. [LHP Collaboration], PoS LAT2008, 141 (2008) arXiv:0810.1933 [hep-lat]].

[28] S. Ohta and T. Yamazaki [for RBC and UKQCD Collaborations and for RBC and UKQCD Collaborations], arXiv:0810.0045 [hep-lat].

[29] T. R. Hemmert and W. Weise, Eur. Phys. J. A 15,487 (2002).

[30] M. Gockeler et. al, Phys. Rev. D 71, 034508 (2005).

[31] T. Yamazaki et al., arXiv:0904.2039 [hep-lat].
[32] C. Alexandrou, G. Koutsou, T. Leontiou, J. W. Negele and A. Tsapalis, Phys. Rev. D 76, 094511 (2007).

[33] T. Yamazaki et al. [RBC+UKQCD Collaboration], Phys. Rev. Lett. 100, 171602 (2008).

[34] T. R. Hemmert, M. Procura and W. Weise, Phys. Rev. D 68, 075009 (2003).

[35] C. Alexandrou et al., Phys. Rev. D 77, 085012 (2008).

[36] V. Pascalutsa and M . Vanderhaeghen, Phys. Rev. Lett. 95, 232001 (2005).

[37] C. Alexandrou et al., Phys. Rev. D 79, 014507 (2009).

[38] M. Burkardt, Phys. Rev. D 62, 071503 (2000) [Erratumibid. D 66, 119903 (2002)]. 